\title{
Author Correction: CYP72A enzymes catalyse 13-hydrolyzation of gibberellins
}

Juan He, Qingwen Chen, Peiyong Xin, Jia Yuan, Yihua Ma, Xuemei Wang, Meimei Xu, Jinfang Chu, Reuben J. Peters (D) and Guodong Wang (iD

Correction to: Nature Plants https://doi.org/10.1038/s41477-019-0511-z, published online 16 September 2019.

In the original version of this Article, "CYP72A9" was incorrectly written as "CPY72A9" in Fig. 7; additionally, the associated legend "CYP72A is highlighted in red" should have read "CYP72A9 is highlighted in red". These errors have now been amended. 